\title{
Wisdom Engineering and the World of Emotion
}

\author{
Shuichi FUKUDA *,** \\ * Stanford University \\ Building 530, 440 Escondido Mall, Stanford, CA, 94305-3030, USA \\ E-mail: shufukuda@cdr.stanford.edu \\ ** Keio University, System Design and Management Research Institute \\ 4-1-1, Hiyoshi, Kohoku-ku, Yokohama, 223-8526, Japan \\ E-mail: shufukuda@gmail.com
}

Received 9 April 2014

\begin{abstract}
This paper proposes we have to go one step further from Knowledge Engineering and develop Wisdom Engineering in order to cope with the very frequently and extensively changing environments and situations. In order to respond quickly and adaptively to such changes, knowledge should be processed more dynamically. Emotion plays an important role for this purpose in sensing and in making decisions. Further, our world is changing from Closed World to Open World. In a Closed World, experience can be accumulated and is structured into knowledge and the logic of induction and deduction can be applied. But in an Open World, decisions must be made by trials and errors by interacting with the outer world. As the fact emotion comes from the Latin word, movere, it literally means to move out or to act toward the outer world. Motivation comes from the same Latin word. Thus, motivation-action-emotion constitutes a cycle and it plays an important role in trials and errors and in producing dynamic knowledge. Wisdom Engineering is as an integration of Emotion Engineering and Knowledge Engineering and it produces dynamic knowledge, which enables us to interact with the frequent and extensive changes of the outer world more wisely and adaptively and to explore the new frontiers in an Open World.
\end{abstract}

Key words : Wisdom Engineering, Emotional Engineering, Open World, Frequent and extensive changes, Trials and errors, Adaptive interaction, Dynamic knowledge, Satisficing

\section{Our World is Changing: From Closed World to Open World}

Our world has been closed with definite boundaries. Thus, set theory held and we could apply such logics as induction and deduction. We could accumulate experience and structure them into knowledge (induction). And we could solve problems by utilizing them (deduction). But our world is quickly expanding and boundaries are disappearing (Fig.1).

When our world is small and closed, there is only one peak and it is apparent that it is the highest. Everybody could find the highest peak without any difficulty. So how more easily and faster we could get to the top of the mountain was our main focus. In other words, tactics played an important role in those days. But when our world becomes larger, there are many peaks and it is not straightforward to identify which peak is the highest. Thus, with the expansion of our world, it becomes increasingly difficult and strategy becomes more important than tactics (Fig.2)

When the world was closed, we could carry out optimization, i.e., we could find the highest peak very rationally. But with the expansion of search space, such local optimization is no more applicable, and we have to introduce global optimization. But unlike local optimization, global optimization is not rational. It is carried out by providing fluctuations and after many trials, the highest one we came across is believed to be the highest. Thus, global optimization is nothing logical at all. It is very much related to emotion as described later in Chapter 3 (Fig. 3 ) 


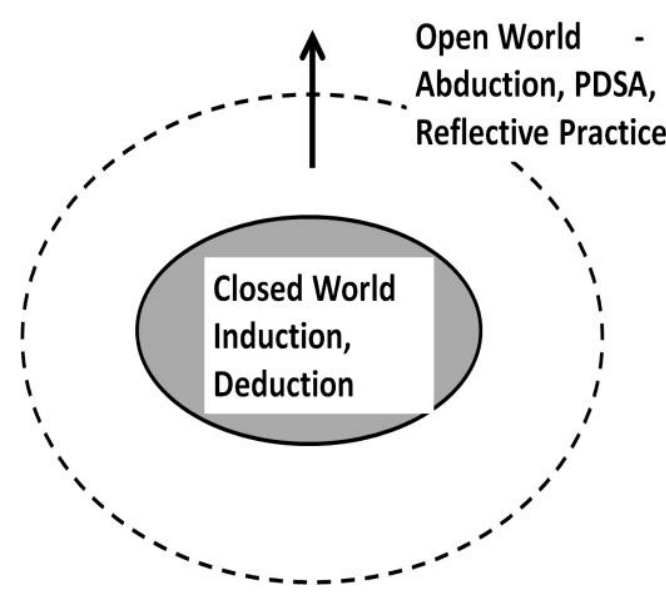

Fig.1 Closed and Open World

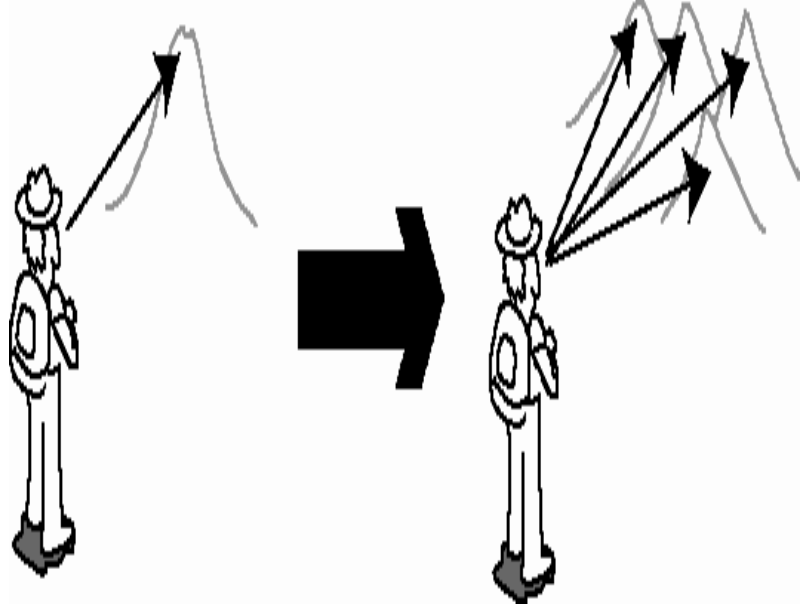

Fig.2 Single Peak to Multiple Peaks

\section{SA Simulated Annealing Global Optimization}

Fluctuations

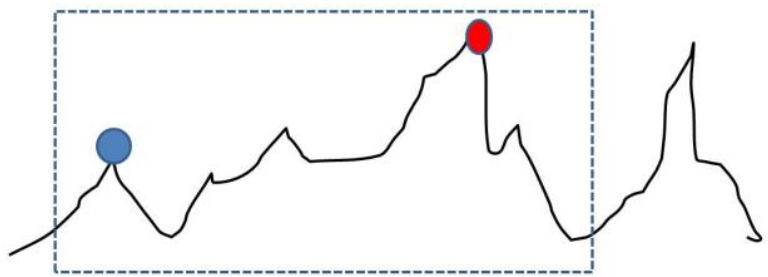

Fig.3 Global Optimization

In addition to its expansion, our world comes to change very often and very extensively. Changes in the past have been smooth, or in mathematical terms "continuous", so that differentiation could be carried out and we could make predictions. But today we come across many bending, or in mathematical terms "discontinuous", changes, which are not differentiable, so that we cannot make any predictions (Fig.4).

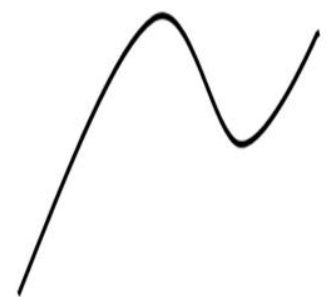

Yesterday

Smooth Change

Differentiable

Predictable

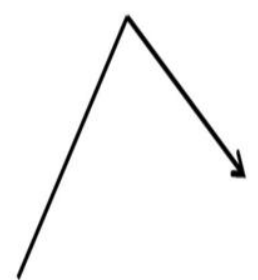

Today

Sharp Change

Not Differentiable

Not Predictable

Fig.4 Continuous and Discontinuous Changes 


\section{Approaches to the Open World}

In a closed world, we could apply rational approaches. But as our world expands into an open world, such traditional, rational approaches no longer hold. We have to look for other approaches.

Abduction ${ }^{1)}$ is one of such approaches. The time Charles Sander Peirce proposed this idea was in the midst of westward movement in the $19^{\text {th }}$ century in the United States. Its fundamental idea is how we can come up with a solution in a new and un-experienced Open World. There are no pieces of prior experience, which will constitute the basis for knowledge. Even if the goal is very clear, we do not know how to get there. Thus, we have to find our way by trials and errors. Abduction in short is to look for a hypothesis or an approach which might be applicable to solve the current problem and observe if it works or not. If it does not, then we look for another one. This is repeated until we come across a successful solution.

PDSA $^{2)}$, proposed by Walter A. Shewhart, expert on experiments of design, is basically the same. This idea is misinterpreted in Japan and is called PDCA (Plan-Do-Check-Act). But this is an approach not to improve the fixed model, but to find a model that adapts to the current situation. So he strongly opposed to his idea being called PDCA. He insisted that his approach should be called PDSA (Plan-Do-Study-Act).

Donald A. Schon published a book "The Reflective Practitioner: How Professionals Think in Action “ 3). He pointed out that in the field of design, management and medicine, there are no straightforward ways or rational ways of stepping forward, so that in these fields professionals step forward by reflecting upon what you did or by thinking in action. It should be noted that the year of publication is 1984. It was the time of great changes so that people could not foresee the future.

What is shared among these three ideas is the philosophy of Pragmatism ${ }^{4)}$. It originated from UK. The essence of Pragmatism can be expressed as "All's well that ends well" 5). What matters most is whether we can achieve our goal or not. How we can get there is marginal compared to getting to the goal.

UK is a famous seafaring country. She conquered the seven seas. On a voyage, you cannot tell what will happen next. After a very clear day, a storm may come up the next day and you may have to change your course. And if the weather is too bad, you may have to give up getting to your original destination and have to find an alternative port that would satisfy what you originally looked for (Fig.5)

\section{Nonlinear Society From Now On}

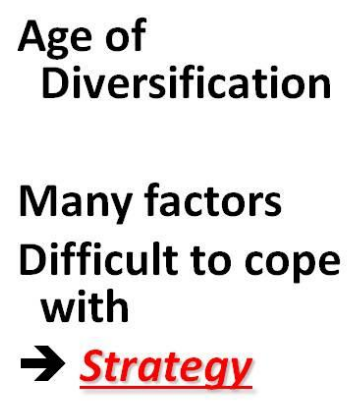

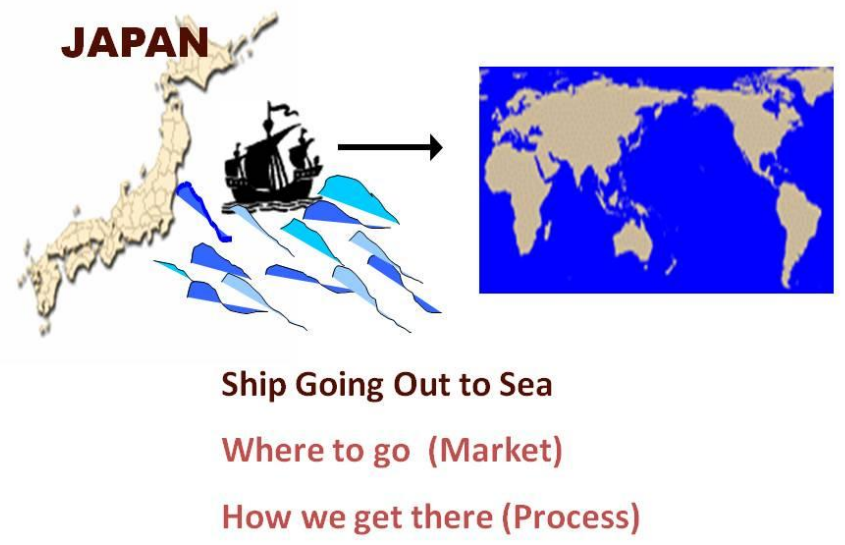

Fig.5 Voyage

US is the country of exploration. She explored the West for gold mines, for example. You may get successfully to a goal mine but when you look back at the desert, the path you followed may most probably be flown away by wind.

Thus, goal finding or strategy is crucial in an Open World. The way or the course to get to your goal depends 
largely upon the situations and you have to get there by trials and errors.

The same situations take place in hunting. In hunting, you cannot tell what animals you may come across. If the one you come across is stronger than you, you might become the prey. It is important to plan what animals you hunt as your game. Thus, goal finding or strategy is crucial in the world of hunting, too (Fig. 6).

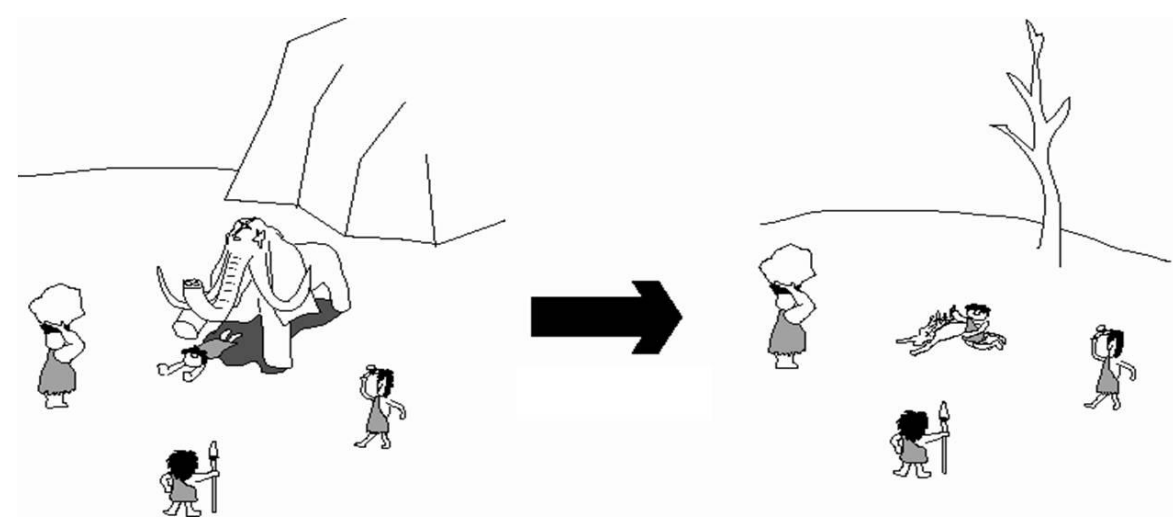

Hunting Type

\section{Creation of New Process and New Market}

Fig.6 Hunting

On the other hand, in the case of railroad, we know which track and train will take us to our destination. The number of choices is limited from the first and how to get there is very much obvious. So what we have to do is to make our decision at an early stage and it is a one-time decision. In the cases of a voyage or hunting, however, decisions have to be made every time adaptively when the situations change. Therefore, it is a multi-stage process. Thus, in the case of a railroad, most of our efforts are paid to make travel faster (Fig.7)

\section{Linear Society \\ Up to Now}
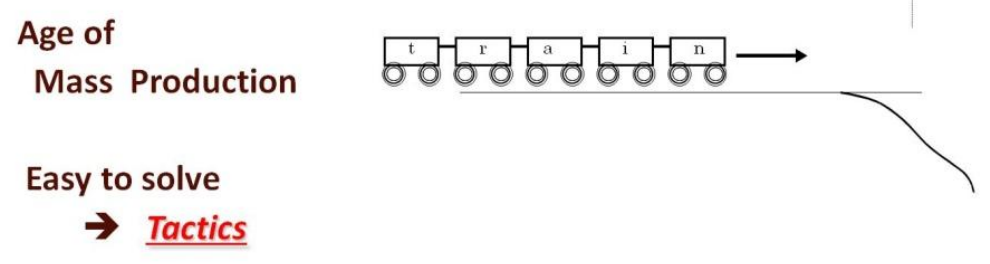

Fig.7 Railroad

The situation is the same in agriculture. The arable land we plow is fixed and bounded. It is a typical Closed World. And we have to make a decision at an early stage what seed we will sow to the land. The more knowledge and experience increase, the better harvest we will reap (Fig.8). 


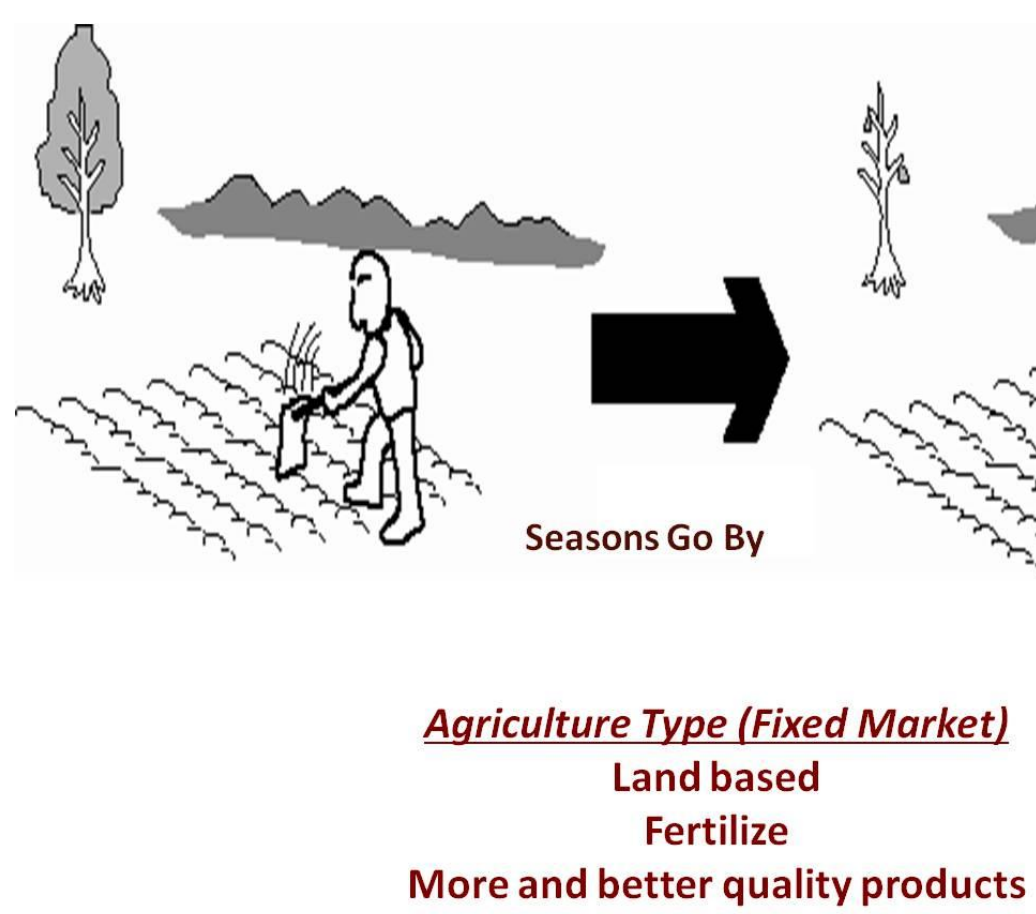

Fig.8 Agriculture

Thus, the Closed World can be compared to the worlds of railroad and agriculture and the Open World to the worlds of voyage and hunting. As they illustrate, rational approaches can be applied in the Closed World, so that we can optimize them. On the other hand, in the Open World, we have to solve the problem by trials and errors and there is no assurance that the goal is the optimum. We have to satisfy ourselves by believing it is the optimum, if we get what we want.

\section{Bounded Rationality and Satisficing}

Herbert A. Simon proposed the idea of bounded rationality ${ }^{6}$. He insisted that our rationality is bounded and beyond such bounds, we resort to emotions. Based on this, he further proposed the idea of satisficing ${ }^{7)}$, a portmanteau of satisfy and sufficing. People accept the result if it satisfies them enough, no matter it may not be optimal. This demonstrates that people make decisions emotionally.

Interestingly enough, John Maynard Keynes, another famous economist, points out that people make decisions rationally for short term expectations, but when it comes to long term, they make their decision based on whether they have confidence or not ${ }^{8}$. This also demonstrates emotion plays an important role in the Open World.

Coming back to the discussion of global optimization, we do not have any guarantee that the result is the optimum. We accept the result because we feel confident that the result is satisfactory enough and it is what we want. Thus, global optimization is none other than satisficing.

\section{Emotion}

Satisficing is an issue of emotion. Emotion comes from the Latin word "e=ex=out and movere=move". Thus emotion literally means to move out. And it should also be remembered that motivation or motive comes from the same Latin word "movere". Thus, emotion constitutes a cycle of motivation-action-emotion as shown in Fig. 9. 


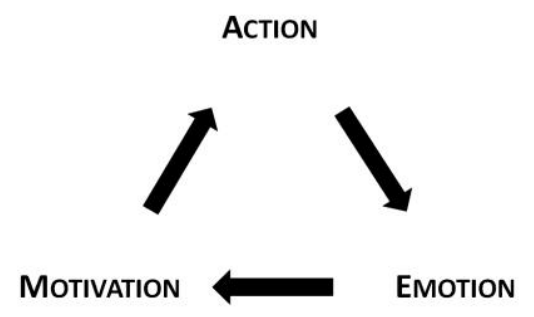

Fig.9 Motivation-Action-Emotion Cycle

Thus, Emotion Engineering 9), 10), 11) is different from Kansei Engineering. In Japan, Kansei is interpreted in a passive sense, like affordance. The line of reasoning is one way from a product to a customer. Emotion Engineering is, however, very much active. A user is motivated and takes an action. Then reaction comes back. A user feels satisfaction or recognizes quality through interactions. It is both ways. Thus, emotion can be described as an interaction with the outer world.

Today, user experience (UX) is getting wide attention because UX is deeply related to quality evaluation. But UX is none other than user's interactions with the outer world or with the situations. Thus, we could say that X in UX, User Experience plays the same role as $\mathrm{x}$ in IxD, Interaction Design. It is interesting to note that $\mathrm{x}$, a saltire or a diagonal cross is observed in numerous flags. It may be interpreted that a crossing or an intersection plays an important role in our life. An active interaction will bring two objects together so that they would form an intersection (Fig.10).
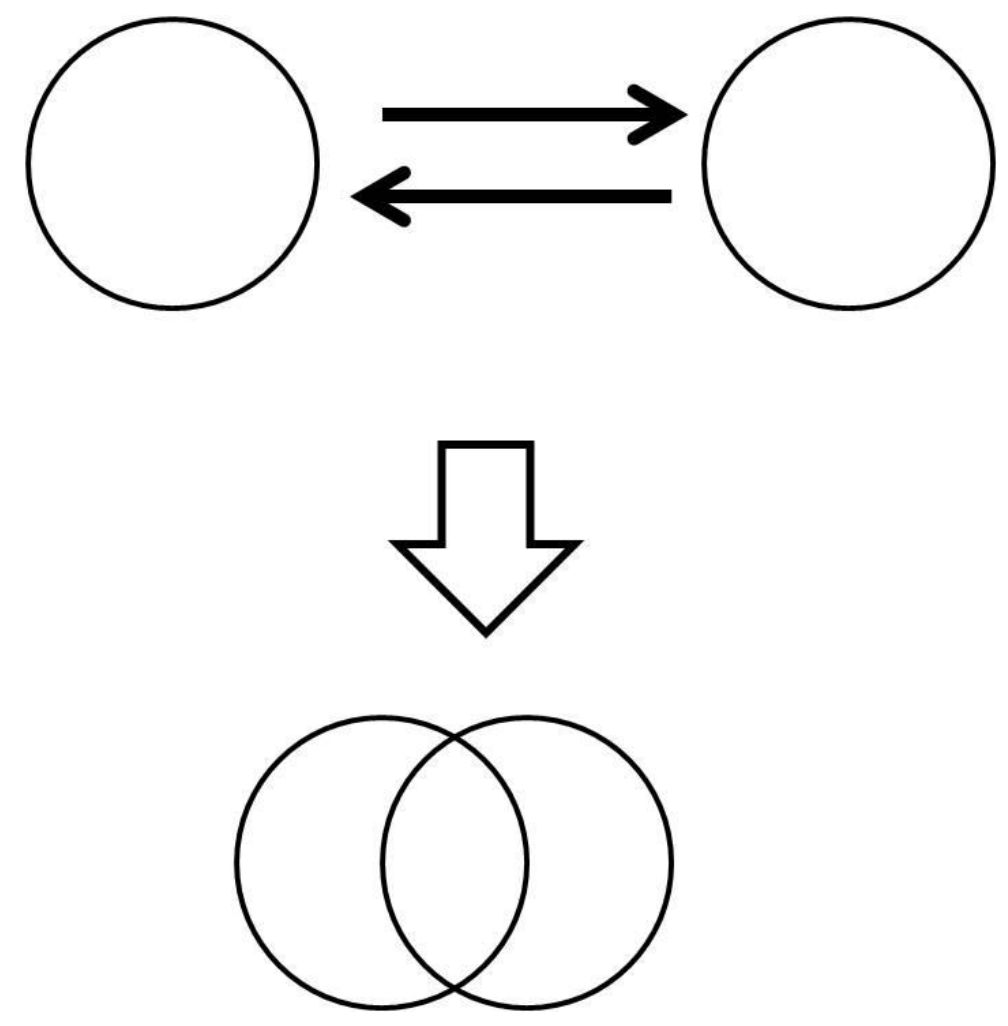

Fig.10 Active Interaction Produces Intersection

Why do we have to interact with the outer world? It is because we have to sense what the environments/situations/contexts are to understand them in order to make an adequate decision for the next step. But in Kansei Engineering, most of which are much more one way, not too much discussions are made about sensing. It should be emphasized that sensing plays a crucial role in Emotional Engineering, which is based on interactions. 


\section{Hardware and Software}

Although such discussions are rarely made, hardware could be thought of an example of the Closed World and software as an example of the Open World. This is because hardware is not only physically bounded, but our knowledge and experience are also bounded. Most of hardware materials are not new to us and we have used them. Thus, we have accumulated experience and we have knowledge about most of hardware objects and materials. Thus, we feel trust in them. Although recently new materials are emerging, but still they are objects in the Closed World so we feel that we can have the same feeling toward them and our concern is much less than software, because they are visible and touchable. We can communicate directly with them. These haptic interactions serve to build up trust in them.

On the other hand, software is non-physical and invisible. And when we buy them, we have never experienced them. Thus, software may be said to be an example of the Open World. In fact, software has no boundaries. We can develop any software as we wish. As it is an object of the Open World, we feel uneasy when we buy them. We are not sure if our expectations will be fulfilled or not.

It is interesting to note how different their developments are between hardware and software. Hardware is developed as shown in Fig.11. It is developed with fixed functions required by design specifications.

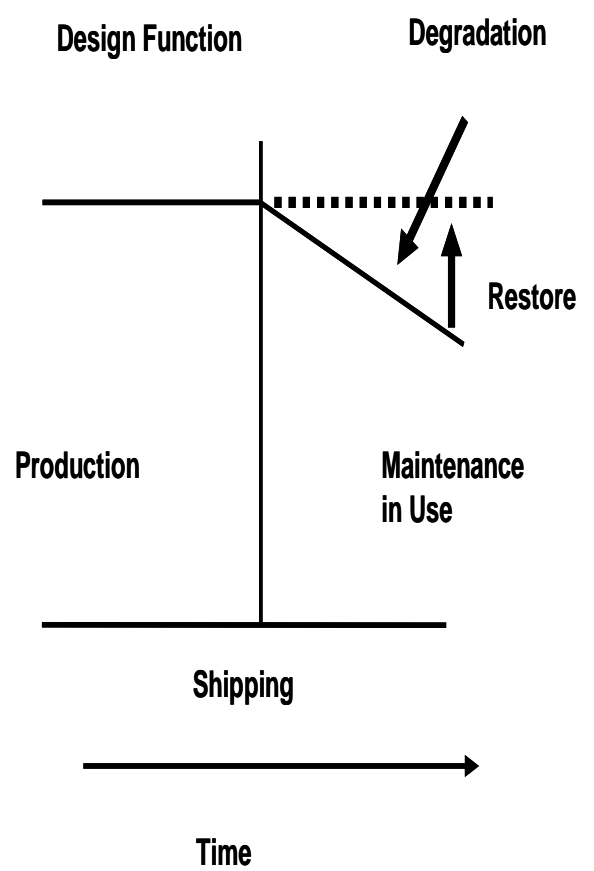

Fig.11 Development of Hardware

Software used to be developed exactly in the same way as hardware. But since it is non-physical, it turned out soon that it is impossible to debug completely or to remove all defects before delivery. And software developers also found out that it is not only difficult to develop software as we do with hardware, but also they cannot convince their customers of their product quality in the same way as hardware.

These findings drove them to introduce another way of development as shown in Fig. 12. The basic functions are provided first and when users get used to this version of a system, functions are upgraded and a little higher level ones are provided. Thus, functions grow or evolve with user experience. This serves to build up trust in these software systems, because when people feel confident in using the system, they put trust in them. Thus, trust increases as user experience increases. 


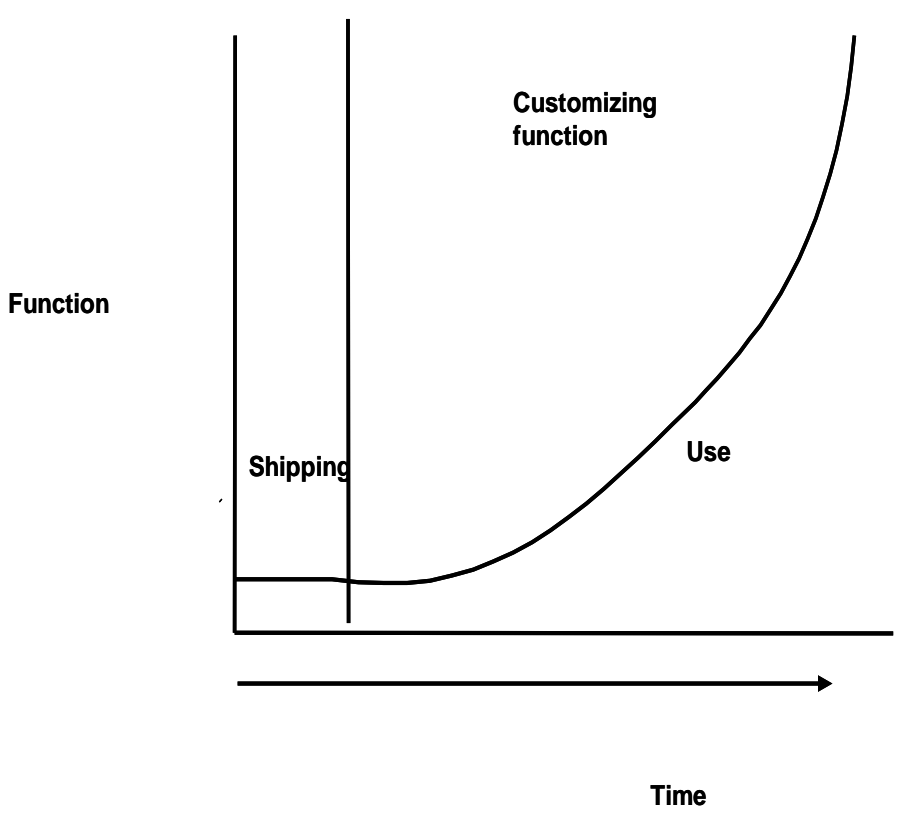

Fig.12 Development of Software

Such continuous prototyping or iterative development served a great deal for reducing the burden on the part of software developers, because they can debug more easily since the search space is very limited. Thus their qualities increased and further as functions are upgraded step by step so that users have time to master them and feel confident, which contributed to building up their trust in the system. Interestingly enough, in German confident and trust are expressed using the same word "Vertrauen". In fact, confidence and trust are deeply related.

The introduction of this new style of growing or evolving function development demonstrates how interactions, i.e., emotions, play an important role.

Its success affected hardware development, too. Hardware was developed mainly with verification on mind so that prototyping used to be just a process to prove that the prototypes satisfy design requirements. But prototyping in hardware development has changed and it is conceived more as a process for validation, whether a developing product satisfies customers.

Thus, even in hardware, prototyping is changing from traditional one-time verification process to continuous and iterative process for validation. This may be attributable to the fact that even hardware products were quickly and increasingly diversifying, so that boundaries are also disappearing quickly even in hardware field.

But although the world of hardware becomes more open than ever before, it should be remembered that the problem of hardware is individual. Its discussion is something like those about John and Smith. Software is not. Its discussion is something like those about human beings. So software essentially belongs to the Open World, while hardware to the Closed World.

In spite of the foregoing discussions, we also have to bear in mind that a wall between hardware and software is quickly collapsing. Hardware used to be analog and software digital.

But emerging technology will be digitalizing hardware. Like Lego, pieces of materials with different attributes will be integrated into a one system or a new material. Our production has been carried out in such a way as material selection-design-manufacturing, because the number of selections of material are limited so that we chose material that would best fit for the purpose and then started design and then developed manufacturing processes that would fit these materials as shown in Fig. 13. 


\section{Material $\square$ Design $\square$ Manufacturing $\square$ Use Selection}

Fig.13 One Way Traditional Production

But when materials are digitalized, this scene will be changed completely. Just like children playing with sand on the beach, materials users want or expect in their daily life could be realized just like software and these functions will be realized. Thus, tomorrow use-material development-design-manufacturing cycle will be repeated during the product lifecycle as shown in Fig. 14.

In other words, production will change from one way to cyclic.

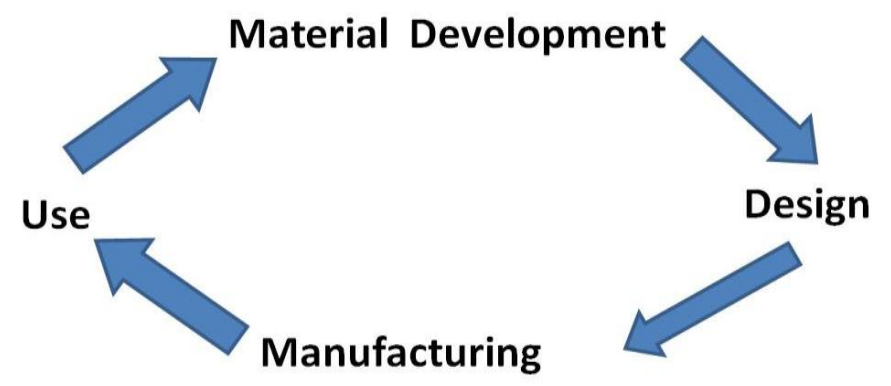

Fig.14 Use-Material Development-Design-Manufacturing Cyclic Production

Thus, in the field of system development and production, the World will become Open so that interactions or emotions will play a crucial role.

\section{Japanese and English}

Consideration about the differences among languages provides another interesting window to the Closed and Open Worlds.

Let us take the Japanese language and the English language for example and compare them.

Nouns play a primary role in Japanese, while verbs do in English.

English has a wide variety of verbs for the same Japanese verb as shown in Fig.15.

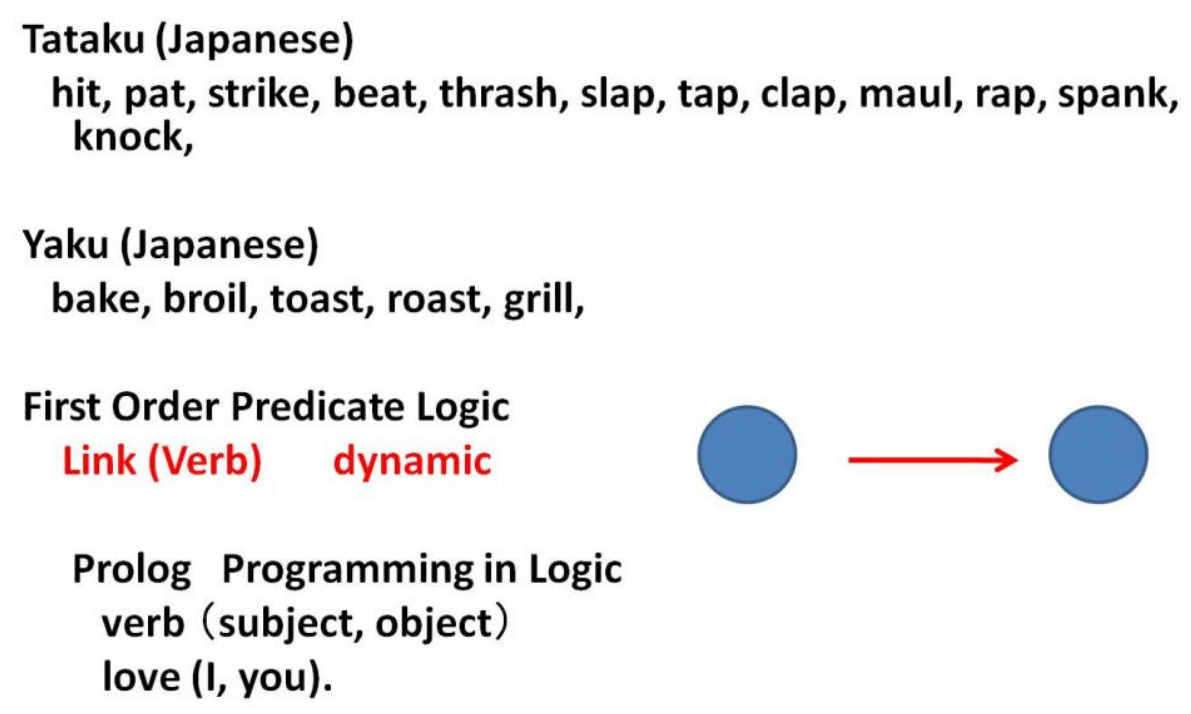

Fig.15 Verbs are Important in English 
This may be deeply associated with the fact that Japanese characters are images. English characters, on the other hand, are phonetic. If you look at the static images of characters, you can tell what they mean. Thus, Japanese is static, while English is dynamic. This may be attributable to the fact that Japan depends chiefly on agriculture, while English is the language of the seafaring UK.

The development of first order logic in Artificial Intelligence and its programming language Prolog demonstrate dynamic feature of English. Using graph theory for explanation, Japanese focuses on nodes, while English does on links. Thus, decision making plays an important role in English. We must decide which verb to use, depending upon the situation. As the verb means action, then once we select one verb, we must select another verb for the next action. Thus each time we must a decision.

On the other hand, Japanese represents a node and understanding Japanese characters is something like pattern recognition. When we come across a Japanese character, we compare it with the pattern of a candidate Japanese character and identify what it is and understand what it means.

Thus, Japanese is the language of one time decision while English is that of the multi-stage decisions. Therefore, it may be said that Japanese is a language of the Closed World, while English is that of the Open World. It is interesting to look at their histories. Japan actually closed her doors to foreigner for more than 200 years from the $17^{\text {th }}$ to $19^{\text {th }}$ century. On the other hand, Great Brain dominated the Seven Seas and US advanced their frontiers.

\section{Quality}

The importance of verbs can be realized if we consider the issue of quality. Quality is what our customer expects from our products or systems. It should be noted that quality is deployed into functions using verbs in Quality Function Deployment (QFD). Thus, quality represented as functions is dynamic. Especially mechanical engineers should remember that their products are evaluated chiefly by their functions. So quality is dynamic to them.

And it should also be remembered that most of other items of quality which may look static are really dynamic. For example, if the surface of a product looks very good, it is evaluated good when we touch them. Thus, even such static-looking qualities are evaluated good after we interact with them. Thus, most of the qualities which are considered to be independent from functions are emotional in the sense that they are evaluated through interactions.

\section{Stories}

In this regard, the importance of stories should be stressed. Donald Norman points out that people do not care the size of a display, if it is a map. But they do care when it comes to seeing the movies. This is because movies have stories. People are drawn in and get excited. Looking at a map provides knowledge, but not emotional excitement.

It is also interesting to know that the highest class version of their chairs by Fritz Hansen, famous Danish chair maker use natural leathers, although their middle class version use artificial leathers and their qualities are reputed to be of the highest quality. Why do they use natural leathers for their highest class? That is because natural leathers have bruises and scratches. They produce stories.

\section{Time Dependent Values}

In most areas of engineering, we have been emphasizing reproducibility. We designed our systems to operate in the same manner, no matter how the situations change or how much time has passed since the beginning of their operations. Thus, functions of a final product at the time of delivery are considered to generate product value.

Of course, such time-independent values are important. But we also have to turn our eyes to time dependent values. The difference between still and moving images is one example. What people expect from time independent properties and time varying properties are different.

When we put on new shoes, we feel awkward, but when we wear them long, they come to fit us better and we feel very happy. Such breaking in is important in our daily goods. But we have to remember that we feel happy and trustworthy when mechanical systems break in.

In most research discussions, deterioration is considered to be the phenomenon to be prevented, but we have to 
remember that such breaking in is associated with the phenomenon of deterioration. And when our systems, which include our daily goods, break in, we feel happy and confident that we can use them as we wish and we put trust in them. Thus, the phenomenon of deterioration should be re-examined from the standpoint of interactions between a user and a system and in terms of emotional evaluation.

And it also should be added that such breaking in generate stories and we are attached more and more to the products. This would change the evaluation of product value from the current one time to life time.

\section{Knowledge Engineering}

It is pointed out in Chapter 6 that English is essentially a dynamic language so that it calls for decision making every time a person intends to use a verb. Knowledge Engineering, which is an area of Artificial Intelligence (AI), demonstrates this feature of English. Knowledge can be represented in Prolog as shown in Fig.16.
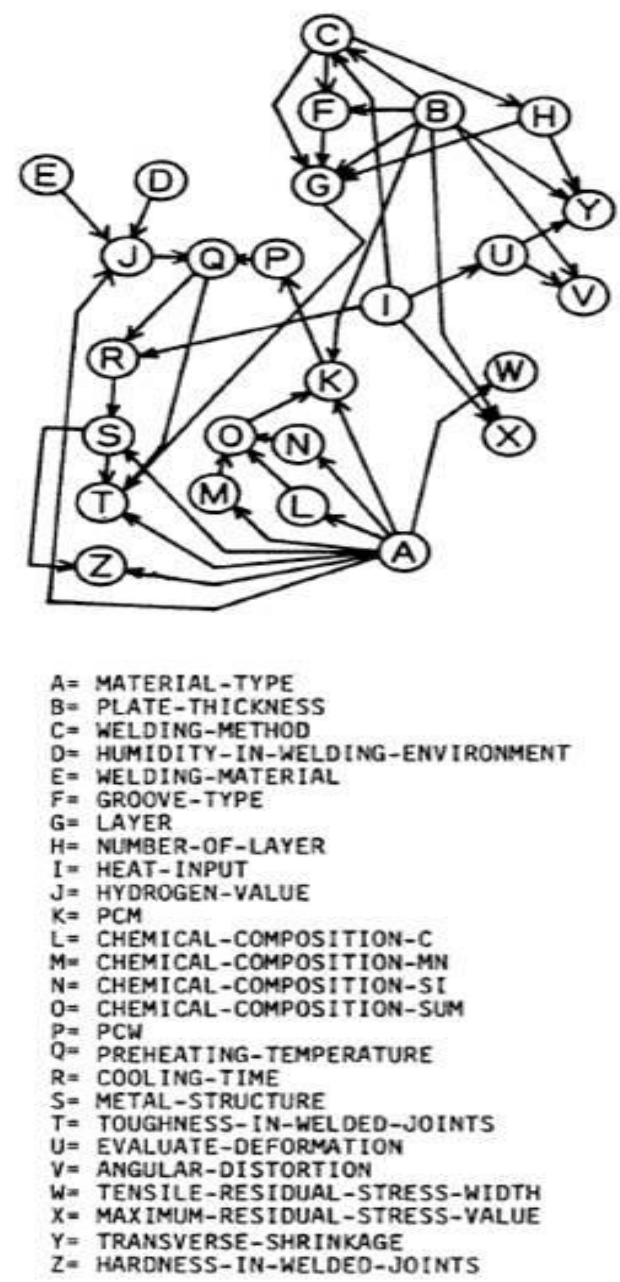

Fig.16 Knowledge Representation

As described earlier, links in the graph represent verbs. And when we come to a branch or a node with multiple links, we have to make a decision which link or action we should take next. In AI, this is carried out by way of pattern matching. The computer looks for the pattern that matches with the fact in hand and select the link. Thus, no matter how much knowledge is entered, the computer can process them. In procedural programming, which had been prevalent until the emergence of AI, we have to make clear the flows of processing. Thus, when a system become large and needs a large amount of knowledge, we can no more develop a useful program. Thus, Knowledge Engineering opened the doors to the Open Word. 
Knowledge Engineering provides a very versatile and useful tool in the age of diversification and complexity. We need many heads from many different fields to solve the problem. But no man can see the whole picture. If there is such a person, he or she can oversee the program development and the task can be described in procedural programming. But in reality, more and more diverse and complex knowledge and experience are called for with the increasing diversity and complexity, we cannot program such a task in procedural programming. Knowledge Engineering based on the idea of declarative programming is a tool for such applications. Even if the diversity of backgrounds of experts increases, experts can build up a graph network by putting their pieces of knowledge and experience into the network. What they have to take care is only the consistency between the neighboring areas. Although it is difficult to understand the areas far away and very different from our own area, a network which is composed of many different pieces of knowledge and experience from many diverse areas can be developed.

Fig. 16 shows an example of how effective Knowledge Engineering is. Although WPS (Welding Procedure Specification) is legally required to perform welding, no one can produce WPS alone, because welding requires a wide variety of knowledge and experience such as material, mechanical. electrical engineering, etc. Further the knowledge and experience of the application field such as civil, shipbuilding engineering, etc are needed to develop WPS for a particular application. But by introducing Knowledge Engineering, we can successfully put them together to produce WPS ${ }^{12)}$

What is very important here is that nobody in the team really sees the whole picture. But each member can offer options which he or she thinks reasonable. But it often happens that even if some options may look reasonable to some members, others may not be able to judge. This is where Knowledge Engineering is very effective. It allows lazy evaluation. Thus, the members who are not sure if the selection of a link is reasonable can postpone their judgments until later nodes when they feel sure that the links selected up to that node are reasonable. Then they can approve the selections made up to that node. If a node provides no adequate links, then we can backtrack to the previous node and take another link (Fig.17).

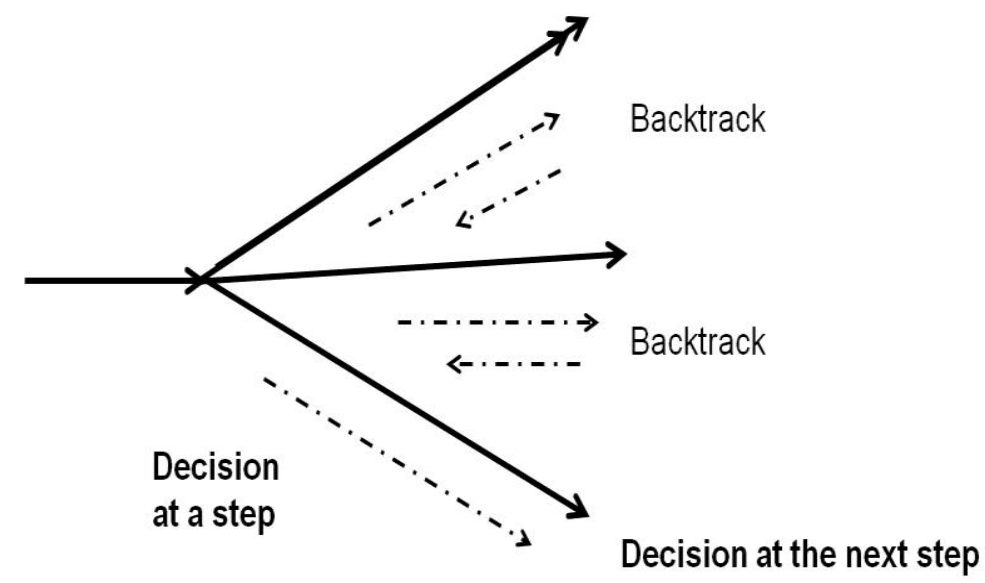

Fig.17 Backtracking

When we cannot find an adequate link after all searches, then we can relax the constraints and repeat the search again.

Thus, Knowledge Engineering is a tool for satisficing. Nobody knows that the solution is the optimum, but everybody is convinced or satisfied enough that the solution is adequate and reasonable.

Now, we have seen how Knowledge Engineering is effective for applications which call for diverse and complex knowledge and how it permits trials and errors. But is this enough to explore the Open World?

No. Then, what is missing? It is sensing. Although Prolog provides a mechanism of pattern matching using verbs, the links to be selected are static. They are programmed for a particular context. But when the environments/situations/contexts change, the piece of knowledge and experience also change in response to their changes. Thus, this knowledge (and experience) network changes with them. Current Knowledge Engineering representations using graphs is static. It does not fully take into consideration that the Open World is dynamic. Knowledge Engineering is still halfway to the Open World and although it deals successfully with the quickly 
expanding boundaries, it still lacks the capabilities to introduce the dynamic nature of the Open World.

\section{Knowledge and Wisdom}

Then, what is the difference between Knowledge and Wisdom?

Knowledge is, as discussed in the previous chapter, static and is applied one way. Our knowledge and experience are accumulated in particular environments/situations/contexts. And it is developed in the world with boundaries, no matter how large the world is. So even if we could develop knowledge of knowledges, or meta-knowledge, it is still within the bounded world.

Wisdom is, on the other hand, dynamic and interaction plays a fundamental role there. Wisdom is, in short, to define the boundaries we can deal with in the Open World, in order to make the most of our knowledge and experience. Or to describe it in another way, Wisdom is how we can build up our own world or to define the problem our way in the Open World. Then, we can see the whole picture.

Again, it may be interesting to see how Japanese and English differ in this aspect.

Although Knowledge is static, Knowledge expressed in English contains the list of actions because verbs play a major role in English. What is lacking there is how we define the problem or how we define our world. The list describes only actions after the problem is defined and fixed. The advantage of Knowledge expressed in English is that once a problem is defined and fixed, then we can utilize our accumulated knowledge in a very dynamic way by introducing trial and error approaches.

Chishiki, Japanese translation of Knowledge, is very much static. Nouns play a primary role there, because nouns are key players in the language. And the way Japanese utilize their Chishiki is to match it with the pattern of a problem. There are very few, if ever, descriptions about the methods of trials and errors. This distinguishing feature also holds true with Chie, Japanese translation of Wisdom. Although Chie is more versatile, the essence is the same. Both solve the problem by pattern matching.

\section{Explicit and Tacit Knowledge}

Michael Polanyi proposed the idea of Explicit and Tacit Knowledge ${ }^{13)}$. Knowledge defined here is his Explicit Knowledge. And Wisdom here is thought to be his Tacit Knowledge.

When we talk about Tacit Knowledge, bike riding is often taken up as an example, because it is very difficult to formulate how to ride a bike. But the problem of sensing is not explicitly discussed in most cases.

Let us consider the problem of sensing from the standpoint of Mind and Brain. It may be said that Knowledge is more closely associated with Brain, while Wisdom is more with Mind.

Brain processes many information coming in, but major emphasis is placed on its knowledge processing. There are not too much discussions about sensing. It certainly processes sensing information, but how to sense the outer world or the situation in order to make a decision is not too much discussed. Brain science is progressing remarkably, but what they are working on is how sensed information is processed in the brain. What should be sensed and how they can be sensed are not discussed at all or very few, if ever. In other words, the major focus of brain science now is on how the brain solves the problem. How we can define a problem is still an open question in brain science.

Mind, on the other hand, is associated with consciousness, perception, thinking, judgment and memory. In short, Mind or a mental activity relates very closely to interaction and sensing. And as the fact that Mind and Body are very often used in pairs demonstrates, they are very much connected. Consciousness is thought to be deeply related to the problem definition or how we build up our own world.

In traditional mechanical engineering, however, we have regarded Body more often as actuators rather than as sensors. But Mind and Body interact with each other and Emotion plays an important role to connect them together and to let them work interactively, as W. Timothy Gallwey pointed out in his book ${ }^{14)}$.

We, mechanical engineers, have to take notice of the important role of our body and how important sensing is to explore the Open World. What characterizes mechanical engineering is that it deals with physical and tangible things. But we seem to forget how our bodies enable interactions. We have to come back to our basics and study how we can communicate with the outer world more interactively, utilizing our somatic system to its full extent in order to build up our own world in the Open World. 
Recently haptics is getting wide attention especially in the field of telegrasping in robotics. In the old days, it is relatively easy to grasp an object from a distance. What make telegrasping difficult today is that objects have diverse attributes and they are getting softer so that until we grasp them, we do not know exactly how they would behave. Telegrasping was easy yesterday, because we can easily identify a model from its images. But today we cannot grasp them unless we do trials and errors. Haptics enables real time direct communication with the object. Image processing is not real time and indirect. Haptics facilitates us to change from fixed to adaptive modeling. It should also be added that Motivation-Action-Emotion cycle plays an important role in telegrasping.

Thus, Emotion is not only important from the standpoint of human centered design, but it opens doors to the Open World. In fact, babies grope around and touch many things. Through these interactions, they build up their models adaptively.

\section{Extended Rationality}

Wisdom may be described as our efforts to extend our rationality or to make the Closed World large by expanding its boundaries.

There are many examples in engineering, where although we cannot predict their behaviors, we utilize them for developing products and systems. Let us take arc for example. There are many researches on arc, but we cannot predict how it will behave. If we could, we could prevent thunder. But although we cannot predict its behavior, we can control its behavior. Thus, although phenomena or objects may defy our rational approaches, they can be controlled for engineering applications. In the case of welding, we know from our experience that we can control it if we identify the parts or points which can be analyzed rationally outside of the welded parts. This can be figuratively explained by taking a river as an example. A river changes its shape all the time. In the old days rivers changed their courses, too. Then why can we identify the river and call it by its name? This is because we look at its surroundings and find out the points that do not change over time. In welding, phase changes from gas to liquid and to solid. Such phase transformation may be controllable, be we cannot predict its behaviors. But if we observe the local points around the welded part which do not change or which are not affected by such transformation, we can control its behavior so that we can build bridges, building, etc.

There are many phenomena or objects which we cannot make any predictions, but we can control for engineering applications. This is a big difference between science and engineering. Engineering has been developed and is being developed upon such Extended Rationality. This may be called Wisdom. And we have to remember that sensing played an important role for developing such an approach.

\section{Challenge to the Real World}

We have to remember that Engineering is a challenge to the Real World. This makes Engineering completely different from Science.

Science, or Natural Science to be exact, is to discover the rules that govern the natural world. But engineering is an activity of creation. It creates things that are not available in nature. In Science, you can do whatever you like in the way as you like to discover. You can build up a laboratory and you can take time as much as you like. Thus, Science is one way in this sense.

But in Engineering, we have to develop artifacts in response to customer's expectations. It is not just a one way activity of creation. Engineering is both ways or interactive. And resources (time, money, etc) are limited. We have to satisfy our customers under these severe restrictions.

Therefore, in order to achieve our goals in engineering, we have to look at the real world and understand it as it is. The following books would open engineers' eyes and would let them realize how important it is to see the world as it is.

Betty Edwards, art educator, wrote "Drawing on the right side of the brain" ${ }^{15)}$. Children draw sketches what they see as they are in their very early years. But as they grow older, they come to draw a sketch based upon a concept. For example, a very young child might draw a car from its front. Then, people would not understand if it is a car or a human face. But older children draw a car viewed from its side, so everybody understands it is a car. Thus, older ones draw sketches based upon their structured knowledge. But very young ones draw them as they see. 
Antoine de Saint-Exupery pointed out the same thing in his book "the Little Prince" ${ }^{16)}$. The prince draws a picture of a snake swallowing an elephant. That is what the little prince saw and that is what he drew. But every grown-ups said "It is a picture of a hat". We, engineers, should consider deeply the meaning of what he said. "Grown-ups never understand anything for themselves, and it is tiresome for children to be always and forever explaining things to them".

\section{References}

(1) http://en.wikipedia.org/wiki/Abductive_reasoning.

(2) https://www.deming.org/theman/theories/pdsacycle.

(3) Donald A. Schon, The Reflective Practitioner: How Professionals Think in Action, (1984) ,Basic Books.

(4) http://en.wikipedia.org/wiki/Pragmatism.

(5) http://en.wikipedia.org/wiki/All's_Well_That_Ends_Well.

(6) http://en.wikipedia.org/wiki/Bounded_rationality.

(7) http://en.wikipedia.org/wiki/Satisficing.

(8) John M. Keynes, The General Theory of Employment, Interest and Money, (1936), Palgrave Macmillan.

(9) Fukuda, S., Emotional Engineering, Vol.1 (2011), Springer.

(10) Fukuda, S., Emotional Engineering, Vol.2 (2013), Springer.

(11) Fukuda, S., Emotional Engineering, Vol.3 (Springer, to appear in 2014).

(12) Fukuda, S., Maeda, A., Kimura, M., Development of an Expert System of Weld Design Support: To Provide Advice on Determination of Weld Conditions to Prevent Weld Cracking in a Pressure Vessel, Transactions of the Japan Society of Mechanical Engineers, Series A, Vol.52, No.476(1986), pp.1183-1190.

(13) Polanyi, M., The Tacit Dimension, (1966), University of Chicago Press.

(14) W. Timothy Gallwey, The Inner Game of Tennis: The Classic Guide to the Mental Side of Peak Performance, (1997), Random House.

(15) Edwards, B., Drawing on the Right Side of the Brain, (1979), Penguin Putnam.

(16) Antoine de Saint-Exupery, The Little Prince, (1943), Reynal \& Hitchcock. 